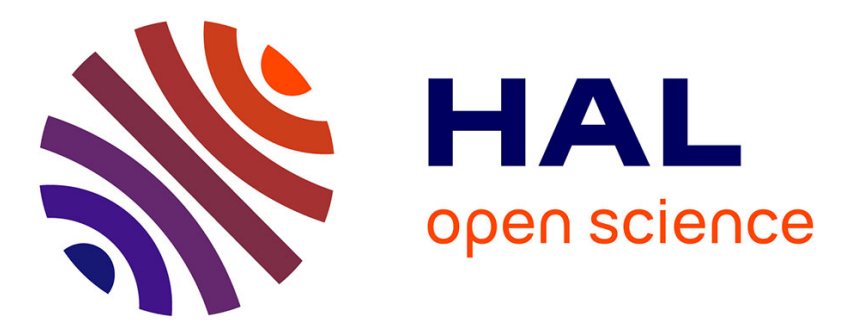

\title{
Nonlinear local parallel acceleration of electrons through Landau trapping by oblique whistler mode waves in the outer radiation belt
}

\author{
O. V. Agapitov, A. V. Artemyev, D. Mourenas, F. S. Mozer, V. \\ Krasnoselskikh
}

\section{- To cite this version:}

O. V. Agapitov, A. V. Artemyev, D. Mourenas, F. S. Mozer, V. Krasnoselskikh. Nonlinear local parallel acceleration of electrons through Landau trapping by oblique whistler mode waves in the outer radiation belt. Geophysical Research Letters, 2015, 42 (23), pp.10,140-10,149. 10.1002/2015GL066887 . insu-01351962

\section{HAL Id: insu-01351962 \\ https://hal-insu.archives-ouvertes.fr/insu-01351962}

Submitted on 13 Sep 2016

HAL is a multi-disciplinary open access archive for the deposit and dissemination of scientific research documents, whether they are published or not. The documents may come from teaching and research institutions in France or abroad, or from public or private research centers.
L'archive ouverte pluridisciplinaire HAL, est destinée au dépôt et à la diffusion de documents scientifiques de niveau recherche, publiés ou non, émanant des établissements d'enseignement et de recherche français ou étrangers, des laboratoires publics ou privés.

\section{(1) (1) $\$$}

Distributed under a Creative Commons Attribution - NonCommercial - NoDerivatives| 4.0 


\section{Nonlinear local parallel acceleration of electrons through Landau trapping by oblique whistler- mode waves in the outer radiation belt}

Article in Geophysical Research Letters · December 2015

DOI: 10.1002/2015GL066887

CITATIONS

3

5 authors, including:

Oleksiy Agapitov

University of California, Berkeley

83 PUBLICATIONS 461 CITATIONS

SEE PROFILE

\section{Krasnoselskikh}

CNRS Orleans Campus

247 PUBLICATIONS 2,255 CITATIONS

SEE PROFILE
READS

98

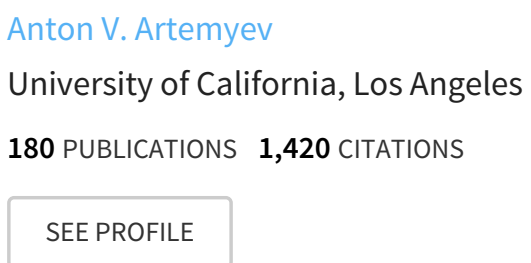

Anton V. Artemyev

180 PUBLICATIONS 1,420 CITATIONS

SEE PROFILE 


\title{
Nonlinear local parallel acceleration of electrons through Landau trapping by oblique whistler-mode waves in the outer radiation belt
}

\author{
O. V. Agapitov ${ }^{1,2}$, A. V. Artemyev ${ }^{3}$, D. Mourenas ${ }^{4}$, F. S. Mozer ${ }^{1}$,
}

V. Krasnoselskikh ${ }^{1,5}$

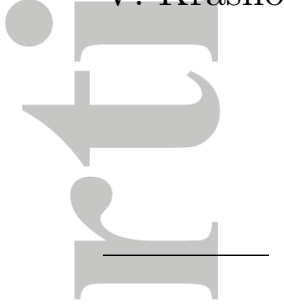

Agapitov O.V., Space Sciences Laboratory, University of California, Berkeley, California, USA

Artemyev A.V., UCLA, Los Angeles, CA, USA

Mozer F.S., Space Sciences Laboratory, University of California, Berkeley, California, USA

Mourenas D., CEA, DAM, DIF, Arpajon, France

Krasnoselskikh V., LPC2E/CNRS-University of Orleans, Orleans, France

${ }^{1}$ Space Sciences Laboratory, University of

California, Berkeley, California, USA.

${ }^{2}$ National Taras Shevchenko University of

Kiev, Kiev, Ukraine.

${ }^{3}$ UCLA, Los Angeles, CA, USA

${ }^{4}$ CEA, DAM, DIF, F-91297, Arpajon,

France.

This article has been accepted for publication and undergone full peer review but has not been through the copyediting, typesetting, pagination and proofreading process, which may lead to differences

between this version and the Version of Record. Please cite this article as doi: 10.1002/2015GL066887

(C)2015 American Geophysical Union. All Rights Reserved. 
Simultaneous observations of electron velocity distributions and chorus waves by the Van Allen Probe B are analyzed to identify long lasting (more than 6 hours) signatures of electron Landau resonant interactions with oblique chorus waves in the outer radiation belt. Such Landau resonant interactions result in the trapping of $\sim 1-10 \mathrm{keV}$ electrons and their acceleration up to 100-300 keV. This kind of process becomes important for oblique whistler-

mode waves having a significant electric field component along the background magnetic field. In the inhomogeneous geomagnetic field, such resonant interactions then lead to the formation of a plateau in the parallel (with respect to the geomagnetic field) velocity distribution due to trapping of electrons into the wave effective potential. We demonstrate that the electron energy corresponding to the observed plateau remains in very good agreement with the energy required for Landau resonant interaction with the simultaneously measured oblique chorus waves over 6 hours and a wide range of $L$ shells (from 4 to 6 ) in the outer belt. The efficient parallel acceleration modifies electron pitch-angle distributions at energies $\sim 50-200 \mathrm{keV}$, allowing us to distinguish the energized population. The observed energy range and the density of accelerated electrons are in reasonable agreement with test particle numerical simulations.

${ }^{5}$ LPC2E/CNRS-University of Orleans, Orleans, France.

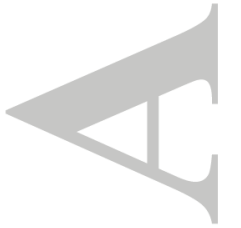

(C)2015 American Geophysical Union. All Rights Reserved. 


\section{Introduction}

Understanding the dynamics and loss of charged particles (and the corresponding evolution of particle fluxes) in the Earth radiation belts is a tremendous task that has already been going on for decades and still remains incomplete. The Van Allen radiation belt particle populations evolve in a dynamic equilibrium between losses (mainly due to particle precipitation to the upper atmosphere or magnetopause shadowing) and re-filling due to external injection, transport, and local acceleration processes. Recent results from the Van Allen Probes have provided additional evidence concerning the local nature of many processes of particle acceleration in the heart of the outer belt [e.g., see Reeves et al.,

2013; Mozer et al., 2015] and it is now generally agreed that such in-situ acceleration mechanisms are very important in this region of space.

A favored candidate for driving in-situ acceleration (and scattering) is the interaction between whistler-mode waves and electrons. In the inner magnetosphere, whistler-mode waves manifest themselves as electromagnetic perturbations generated near the geomagnetic equator and called chorus (structured, coherent, wave packets) and hiss (mostly diffusive, wide band emissions with randomized phases but also sometimes observed as structured emissions [Summers et al., 2014]). Chorus waves are the most intense natural VLF electromagnetic emissions in the magnetosphere, with maximum amplitudes (up to 2 $\mathrm{nT}$ in magnetic field and up to $300 \mathrm{mV} / \mathrm{m}$ in electric field) located in the core of the outer Van Allen radiation belt [e.g., Agapitov et al., 2013, and references therein]. This fact, as well as the closeness of their frequency to the electron gyrofrequency, makes them one of the key factors that control the dynamics of the outer radiation belt [Thorne et al., 2010; (C)2015 American Geophysical Union. All Rights Reserved. 
Horne et al., 2005; Reeves et al., 2013]. Interactions between whistler-mode waves and electrons are usually analyzed in the frame of two different approximations. The quasilinear approach, corresponding to a slow particle diffusion in momentum space, can be used for broadband waves of relatively moderate amplitudes. It was developed by Kennel and Petschek [1966]; Trakhtengerts [1966], and applied to the radiation belts by Lyons et al. [1972] with inclusion of particle diffusion in both energy and pitch-angle spaces [Mourenas et al., 2014a; Albert, 2007; Shprits et al., 2007; Glauert and Horne, 2005] as well as radial transport of electrons from and to the magnetopause[Schulz and Lanzerotti, 1974]. The non-linear approach (i.e. the second type of approximation) describes particle trapping into the effective potential of large amplitude coherent waves with a characteristic interaction timescale about a fraction of the bounce period. The interaction of electrons with coherent whistler-mode waves and their resulting acceleration have been extensively studied for non-linear cyclotron resonance between a parallel propagating wave and oppositely moving electrons [Bortnik et al., 2008; Omura et al., 2007; Albert, 2002]; this process is now well understood analytically as well as through numerical simulations [see the review by Shklyar and Matsumoto, 2009], although corresponding observations are still lacking.

Moreover, while the ray path of chorus waves tends to follow a geomagnetic field line, their wave normal angle $\theta$ can deviate substantially from the background magnetic field direction along their propagation to higher latitudes [Agapitov et al., 2013]. Such a deviation from the parallel direction provides additional effects for the quasi-linear scattering and acceleration of electrons [Mourenas et al., 2014a] and it opens the door for an alterna-

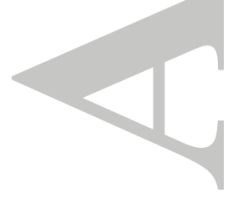

(C)2015 American Geophysical Union. All Rights Reserved. 
tive mechanism of nonlinear interaction: electron trapping into the effective potential of oblique whistler-mode waves through the Landau resonance [e.g., see Bell, 1986; Shklyar and Matsumoto, 2009; Artemyev et al., 2012; Nunn, 1971]. Landau(cyclotron) resonance corresponds to a condition $\omega+n \Omega_{c e}=k_{\|} V_{\|}$with $n=0(-1)$, where $\omega=2 \pi f$ is the wave frequency, $\Omega_{c e}=2 \pi f_{c e}$ is the local electron cyclotron frequency, $k_{\|}$and $V_{\|}$are the parallel components of the wave vector and electron velocity, respectively. The discovery of whistler-mode waves of large electric field amplitudes above $100 \mathrm{mV} / \mathrm{m}$ [Wilson et al., 2011; Agapitov et al., 2014a; Kellogg et al., 2011; Cully et al., 2008; Cattell et al., 2008] has caused renewed interest for non-linear effects in wave-particle interactions. Additionally,

it was shown that a significant portion of the observed high-amplitude waves were very oblique [Agapitov et al., 2014a]. The large parallel electric field component of oblique waves (up to $300 \mathrm{mV} / \mathrm{m}$ ) allows them to interact efficiently with electrons via Landau resonance, sometimes more efficiently than via cyclotron resonance when $\theta$ is larger than the local Gendrin angle $\theta_{G}=\arccos \left(2 \omega / \Omega_{c e}\right)$ [Gendrin, 1968]. When considering Landau resonance, the energy threshold for nonlinear trapping is reduced down to $\sim 1 \mathrm{keV}$ only [Artemyev et al., 2012; Agapitov et al., 2014a], potentially leading to rapid parallel electron acceleration up to $100-200 \mathrm{keV}$ from one trapping event, and even more (up to $0.5 \mathrm{MeV}$ ) for multiple trapping events [Artemyev et al., 2012]. Landau resonant trapping can provide a significant feedback to the wave field, particularly for its parallel electric field component which can be steepened due to the modulation of resonant electron beams [Drake et al., 2015]. These effects were detected in electric field measurements

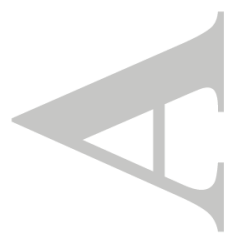

(C)2015 American Geophysical Union. All Rights Reserved. 
from STEREO [Kellogg et al., 2010] and in Van Allen Probes measurements in the outer radiation belt [Mozer et al., 2014].

Statistical signatures of electron Landau resonant interactions with chorus waves in the form of local minima in the derivative of the parallel velocity distribution near the Landau resonant velocity were previously identified from simultaneous in situ wave and particle observations on THEMIS [Min et al., 2014]. Nevertheless, these two quantities differed significantly in a substantial portion of the selected events, while no minimum of the derivative at all was found in $20 \%$ of the cases. This could have stemmed from a too low parallel wave electric field in the considered events or from an insufficient precision of measurements, in particular as regards the plasma density [Min et al., 2014]. Here we provide, for the first time, clear evidence of Landau resonance interaction from a longlasting event during which the location of the plateau in the electron distribution varied strongly and in full accordance with simultaneous changes in the parallel velocity for Landau resonance deduced from precise wave and plasma density measurements from the Van Allen Probes. Furthermore, the theoretically expected simultaneous acceleration of electrons through Landau resonant trapping had not been detected before, mostly because of the low probability of trapping events. In the present paper, we show a more than sixhour interval of Van Allen Probe B measurements of oblique to very oblique whistler-mode waves, intense enough to produce such a significant parallel acceleration of the electron distribution. Due to their interaction with the wave parallel electric field, electrons are indeed trapped and accelerated along the background magnetic field, constituting two populations with field aligned fluxes: one $(1-10 \mathrm{keV})$ corresponds to the energy location

(C)2015 American Geophysical Union. All Rights Reserved. 
of the Landau resonance and the other one $(50-200 \mathrm{keV})$ is produced by acceleration in the inhomogeneous dipole geomagnetic field along field lines. The main idea of this acceleration mechanism corresponds to trapped electron motion at the wave phase velocity along a magnetic field of growing strength as the particle moves away from the equatorial plane. In this case, the particle energy changes due to variations of the wave phase velocity and to a combination of conservation of magnetic moment and increase of magnetic field strength (i.e. betatron-like acceleration) [see a detailed description of both mechanisms in Shklyar and Matsumoto, 2009]. Corresponding test particle simulations are provided, fully confirming the experimental results.

\section{Observations}

The Van Allen Probes A and B were launched on August 30, 2012, aiming at studying the radiation belts with unprecedented detail. Here we examine a particular time interval from 15:00 UT (Universal Time) to 22:00 UT on November 1, 2012. We use data provided by the following instruments onboard the Van Allen Probes: the Electric Field and Waves

(EFW) instrument [Wygant et al., 2013] for electric field waveforms; the Electric and Magnetic Field Instrument Suite and Integrated Science (EMFISIS) [Kletzing et al., 2013] for electric and magnetic spectral and polarization information, the DC magnetic field data and electron cyclotron frequency $f_{c e}$, the electron plasma frequency value $f_{p e}$ (deduced from the upper hybrid frequency); the Energetic Particle, Composition, and Thermal Plasma (ECT) instrument [Spence et al., 2013] data of the Helium Oxygen Proton Electron (HOPE) detector for electron fluxes below $50 \mathrm{keV}$ [Funsten et al., 2013], and the Magnetic Electron Ion Spectrometer (MagEIS) $30 \mathrm{keV}$ to $4 \mathrm{MeV}$ measurements [Blake et al., 2013]. (C)2015 American Geophysical Union. All Rights Reserved. 
The waveform data was transmitted at 16,384 samples/s (35,000 from EMFISIS) for 5 s continuous intervals in the burst mode (triggered automatically by large amplitude signals), as well as continuously every $6 \mathrm{~s}$ under the form of full spectral matrices for 65 frequencies (logarithmically spaced from $3 \mathrm{~Hz}$ to $16 \mathrm{kHz}$ for EMFISIS).

We present results based on an analysis of waveform and spectral data captured by Van Allen Probe B on November 1, 2012, consisting of about 80 burst-mode intervals collected over 15:30 16:30 UT and about 30 intervals during 21:00-22:00 UT. During that period, the Van Allen Probe B escaped from the plasmasphere at 14:50 UT $(L=3.2)$, reached its apogee at 16:40 UT ( $L=5.6)$, and crossed back the plasmapause at 23:10 UT $(L=2.9)$. It

was located in the morning sector (from 02:30 MLT at 15:00 UT to 10:00 MLT at 22:00 UT) in the vicinity of the geomagnetic equator (the magnetic latitude $\lambda$ varying from $-5.5^{\circ}$ to $-15.6^{\circ}$, see Fig. 1). Note that the same interval has been previously studied by Cattell C. A. [2015], who reported the presence of chorus waves of anomalously low frequency between the local lower hybrid frequency and $0.1 f_{c e}$ near the spacecraft apogee from $~ 17$ UT to 20 UT. A moderate geomagnetic storm was taking place on November 1, caused by a strong jump of the solar wind pressure from $\sim 1.5 \mathrm{nPa}$ up to $\sim 8 \mathrm{nPa}$ (solar wind speed increasing from $280 \mathrm{~km} / \mathrm{s}$ to $350 \mathrm{~km} / \mathrm{s}$ ), with a sudden commencement at $\sim 15: 30$ UT on October 31 and the beginning of the main phase at $\sim 6$ UT of November 1. The minimal values of $D_{s t}$ were $\sim-60 \mathrm{nT}(-63 \mathrm{nT}$ at 21:00 UT).

Fig. 1a,b shows the electric and magnetic field spectra collected aboard Van Allen Probe B. Large amplitude whistler-mode waves (reaching more than $1 \mathrm{nT}$ ) have been recorded during the whole period spent by the spacecraft outside the plasmasphere. Their

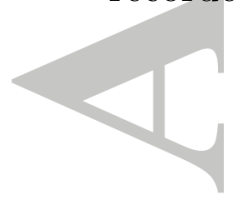

(C)2015 American Geophysical Union. All Rights Reserved. 
Poynting flux is consistent with a generation at the geomagnetic equator. The observed waves mainly lie in the lower-band chorus frequency range $\left(0.1 f_{c e}<f<0.5 f_{c e}\right)$ and are often very oblique. The angle $\theta$ between the wave normal and the background magnetic field direction is displayed in Fig.1c: it generally remains larger than $40^{\circ}$ for $f \geq 0.1 f_{c e}$. Actually, a significant part of the whistler-mode wave power is distributed around the local resonance cone angle $\theta_{r} \sim \arccos \left(f / f_{c e}\right)$. The electric field of these oblique waves is very large (up to $150 \mathrm{mV} / \mathrm{m}$ for a corresponding magnetic field amplitude $\sim 150 \mathrm{pT}$, see Fig.1df). Such wave amplitudes substantially exceed the level required for nonlinear interactions, corresponding to the presence of closed electron trajectories in the phase plane $[$ Bell, 1986;

Albert, 2002; Shklyar and Matsumoto, 2009; Omura et al., 2007; Artemyev et al., 2014b]. Therefore, electron trapping by these waves has to be considered.

Electron trapping into Landau resonance can occur when the parallel particle speed is close to the parallel component of the phase velocity of the wave. Using the cold plasma dispersion relation and considering a wave power spectrum sufficiently peaked at a frequency $f$ and wave normal angle $\theta$, it corresponds to a well-defined parallel kinetic energy for resonant particles [e.g., see Min et al., 2014]:

$$
\varepsilon_{\|}=\frac{m_{e} c^{2}}{2} \frac{f_{c e}^{2}}{f_{p e}^{2}} \frac{f}{f_{c e}}\left(\cos \theta-\frac{f}{f_{c e}}\right) \sec ^{2} \theta
$$

Trapping results in a modification of the distribution function around the parallel energy value given by equation (1). Generally, trapped particles can generate a beam which should be rapidly relaxed to plateau [see discussion in Mourenas et al., 2015]. For a narrow wave power spectrum, the width $\Delta \varepsilon_{\|}$of this plateau is equal to the nonlinear (C)2015 American Geophysical Union. All Rights Reserved. 
resonance width determined by the trapping frequency in the electric field $E_{w}$ of the wave and given approximately (at low energy $\varepsilon_{\|} \ll 1 \mathrm{MeV}$ ) by:

$$
\left(\varepsilon_{\|}+\Delta \varepsilon_{\|}\right)^{\frac{1}{2}}-\varepsilon_{\|}^{\frac{1}{2}}=2\left(\frac{f_{c e}}{2 f_{p e}} \frac{E_{w}}{c B_{0}}\right)^{\frac{1}{2}}\left(\frac{f_{c e}}{f} \cos \theta-1\right)^{\frac{1}{4}}
$$

For the particular wave event considered in Fig. 1g-j, this trapping width $\Delta \varepsilon_{\|}$from (2) is about $1 \mathrm{keV}$. However, a significant additional widening of the Landau resonance and $\Delta \varepsilon_{\|}$generally occurs due to the finite frequency and $\theta$ widths of the actual wave power distribution (see Fig. 1c,i). The effects of Landau trapping can be clearly seen in the distribution of parallel electron flux around 16:00 UT plotted in Fig. 1i. The plateau in the electron distribution turns out to be located precisely in the energy range $\varepsilon_{\|} \sim 10-20$ $\mathrm{keV}$ (highlighted in red) determined from the Landau resonance condition (1) making use of the full distribution of wave power in frequency and wave normal angle $\theta$ presented in Fig. $1 \mathrm{~g}$-h, with average values $B_{w} \sim 100 \mathrm{pT}, f / f_{c e} \sim 0.22-0.25,\left(\theta_{r}-\theta\right)$ is in the range $\left[3^{\circ}, 7^{\circ}\right], f_{p e} / f_{c e} \sim 2$.

The field-aligned to transverse flux ratio is also displayed in Fig. 1j. The highest levels (close to 1) are only observed in the energy range corresponding to strong Landau resonant trapping, demonstrating that the trapped population of electrons at $10-20$ $\mathrm{keV}$ is considerably more field aligned than the background population. This is a direct consequence of Landau resonant trapping: as waves and particles propagate from the equator along magnetic field lines into regions with stronger geomagnetic field, electrons can get trapped by the parallel electric field of the waves and significantly accelerated in this direction, producing a more field aligned population over a range of energy determined (C)2015 American Geophysical Union. All Rights Reserved. 
by the wave amplitude and wave normal angle [this mechanism was considered in details analytically and numerically in Artemyev et al., 2012; Artemyev et al., 2013a, 2014c]. The acceleration process lasts merely a quarter of the electron bounce period and, thus, cannot be observed directly live. Nevertheless, the effects of this acceleration can be discerned in the electron distribution function. An accelerated electron population can indeed be detected around $\sim 200 \mathrm{keV}$ in $1 \mathrm{~h}$ by the considerably larger field-aligned to transverse flux ratio than at $50-100 \mathrm{keV}$ or $500-600 \mathrm{keV}$, as well as by a weak inflection in the profile of the flux distribution - although comparisons with the flux profile recorded just before this event as well as with test-particle simulations will be necessary to ascertain it (see further below). Interestingly, a secondary weaker inflexion occurs near $1.5 \mathrm{keV}$ in the electron flux profile. It likely corresponds to field aligned acceleration through interaction with time domain structures [Mozer et al., 2015, 2014; Vasko et al., 2015; Artemyev et al., 2014a] which occurred at 17 - 19 UT according to low frequency electrostatic noise observed by EMFISIS [Malaspina et al., 2014] (waveforms with TDS were captured by Van Allen Probe A at $\sim 18 \mathrm{UT}$ but are not shown here).

Next, the ratio of field aligned over transverse electron fluxes and the ratio of parallel electron fluxes in neighboring energy channels are displayed as a function of time and energy in Figure 2, respectively in panels a and b. The coincidence of the results in these two different panels over the course of 5 hours demonstrates that the most field aligned electron populations always correspond to the energy range of the plateaus in the parallel flux profiles (indicated by values greater than 1 in Fig.2b), in spite of the strong variation of the plasma parameters along the trajectory of Van Allen Probe B. Indeed,

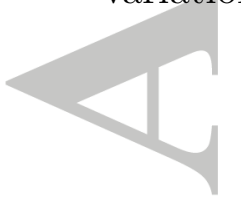

(C)2015 American Geophysical Union. All Rights Reserved. 
the local gyrofrequency $f_{c e}$ (indicated in 1a,b) decreased tenfold from the plasmapause to the apogee while the ratio $f_{p e} / f_{c e}$ increased from $\sim 1$ at 15:15 UT to $\sim 4$ at 18:45 UT, going back down to $\sim 1.5$ at 21:30 UT (the electron density varied from $\sim 20$ $\mathrm{cm}^{-3}$ near the plasmapause to $\sim 2-3 \mathrm{~cm}^{-3}$ at the apogee). More importantly, the energy ranges corresponding to Landau resonance trapping directly calculated from the measured wave frequencies, wave normal angles, and plasma density and geomagnetic field strength measured in situ are displayed in Fig. 2c in the same format as before (with the contributing wave frequencies denoted by different colors). The overall agreement between the three panels in Fig. 2 is striking: the energy range of Landau resonance remarkably matches the energies of plateau and field aligned fluxes over almost 6 hours of measurements from Van Allen Probe B, despite the large variations of all the wave and plasma parameters. This provides a clear and direct evidence of both the presence and importance of Landau resonant trapping in the radiation belts.

Let us now consider in more details the energy range $E \geq 100 \mathrm{keV}$ of the accelerated electron population. Because of the much smaller number of strongly accelerated particles than in the trapped plateau population, this parallel-accelerated population of electrons can be better identified through the modification of the pitch angle distribution that it entails - i.e. by the increase in field aligned fluxes. While the background pitch angle distribution in the MagEIS energy range (from $30 \mathrm{keV}$ to $2 \mathrm{MeV}$ ) has initially a field aligned to transverse flux ratio smaller than 0.1 in general, the presence of an accelerated population after a prolonged burst of oblique chorus waves increases this ratio up to $0.5-0.8$ (see Fig. 3). It corresponds to an increase of the density of electrons in the same (C)2015 American Geophysical Union. All Rights Reserved. 
energy range by $30-50 \%$ through the rapid (in about one bounce period) local parallel acceleration of electrons. Some of the electrons accelerated this way, with pitch-angles simultaneously shifted toward the loss cone, could ultimately be precipitated into the ionosphere, causing microbursts.

The energy gained by electrons through their trapping into the wave effective potential and their subsequent acceleration in the inhomogeneous background magnetic field has been estimated numerically by means of test particle simulations [see detail of wave models and trajectory equations in Artemyev et al., 2014b]. For each time interval (shown in Fig.3a,b,c), we model the wave $\theta$ and frequency distribution by 100 monochromatic waves: ten different frequencies $f=f_{m} \pm 100 \mathrm{~Hz} \cdot m$ with $m=1 . .5$ and $10 \theta$ values for each frequency. The wave amplitude and occurrence rate of each wave corresponds to the distributions collected from EMFISIS waveforms measurements on Van Allen Probe B (see Fig. 3d-i). The considered $\theta$ range is limited to very oblique waves, which are known to possess the largest electric field components [e.g., see Agapitov et al., 2014a; Artemyev et al., 2015, and references therein]: $\theta=\theta_{r}-0.5^{\circ} n$ with $n=1-10$. For each wave, we numerically integrate $10^{5}$ electron trajectories with different initial energies (within the range $1-35 \mathrm{keV}$ ) and pitch-angles (within the range $10^{\circ}-90^{\circ}$ ) in a curvature-free dipolar geomagnetic field model [see details in Artemyev et al., 2014b]. Each test particle is given an effective weight corresponding to its energy and pitch-angle according to the measured energy spectrum of particles during the immediately preceding time period. Trajectories are integrated during a time interval equal to quarter of the bounce period, i.e. only one event of wave-particle resonant interaction is considered (waves are assumed

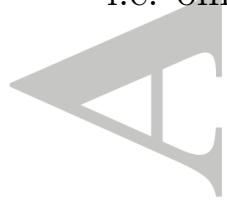

(C)2015 American Geophysical Union. All Rights Reserved. 
to be present up to $37^{\circ}$ degree of magnetic latitude with an effective latitudinal amplitude profile taken from a statistical model presented in Artemyev et al. [2012]). In the end, we collect the final energy distributions for parallel (with equatorial pitch-angles $<25^{\circ}$ ) and perpendicular (with equatorial pitch-angles within the range $75^{\circ}-105^{\circ}$ ) particles and plot the ratio of the corresponding fluxes. The measured wave occurrence rates are used to account for the different contributions of the different waves, i.e. the final energy distribution of accelerated particles is the sum of partial (for each wave) distributions multiplied by the corresponding occurrence rate.

The field aligned to transverse electron flux ratios obtained from test particle simulations are displayed in Fig.3a,b, and c respectively for three different times: 15:45 UT, 15:58 UT, and 16:15 UT, showing a reasonable agreement with corresponding MagEIS levels. The wave distributions used in numerical simulations are displayed in Fig. $3 \mathrm{~d}-\mathrm{f}$ in the $\left(B_{w}\right)-\left(\theta-\theta_{r}\right)$ domain and in Fig.3g-i under the form of a probability distribution function as a function of $\left(\theta-\theta_{r}\right)$, demonstrating the significant presence of very oblique whistlermode waves during the processed intervals - more than $3 \%$ of the observed waves had $\theta$ less than $1^{\circ}$ away from $\theta_{r}$. The large amplitudes of magnetic field perturbations are consistent with observed parallel electric field amplitudes up to $100-150 \mathrm{mV} / \mathrm{m}$. Finally,

Fig.3j presents the processed flux ratio obtained from MagEIS pitch-angle distributions over more than two hours. Local maxima of the flux ratio are observed at energies varying from $\sim 250 \mathrm{keV}$ at 15:00 UT down to $\sim 50 \mathrm{keV}$ at 17:30 UT. Maxima obtained from corresponding numerical simulations are shown by circles filled with colors according to the maximum value of the ratio. The very good quantitative agreement between Van

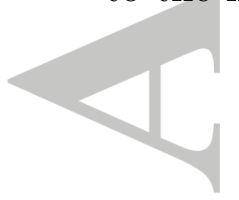

(C)2015 American Geophysical Union. All Rights Reserved. 
Allen Probe observations of $70-200 \mathrm{keV}$ parallel electron flux enhancements and numerical results based on simultaneous wave measurements over more than two hours (UT) and over a significant range of MLT (from 3:30 to 5:30 MLT) and $L$-shells ( $L=4-5.5$ ) demonstrates the very likely presence on that day of a long lasting sequence of nonlinear accelerations of electrons through Landau trapping in the Earth's inhomogeneous magnetic field.

Both the energy range $(70-200 \mathrm{keV})$ and the magnitude of the main increase of the parallel to transverse flux ratio during wave bursts (as compared with its minimum level 5-10 min before) are well recovered in numerical simulations based on Landau resonant trapping (see Fig.3a,b, and c). It is worth noting that an alternative acceleration mechanism via cyclotron resonance with the same very oblique waves measured at $15: 00-16: 15$ UT would require initial electron energies of $50-100 \mathrm{keV}$. However, cyclotron resonant acceleration has been shown to be significantly less effective than Landau resonant acceleration at such low initial energies [Artemyev et al., 2013a], justifying our focus on Landau trapping. Moreover, the Landau resonant acceleration mechanism can fully explain the variation of the energy position of the first maximum of parallel to transverse flux ratio observed in the range 50-200 keV between 15 UT and 17 UT in Fig. 3j. In particular, the energy of this first maximum diminishes as $f / f_{c e}$ decreases (see Figs. 1a and $3 \mathrm{j}$ ), in stark contrast with the simultaneous increase of the minimum energy for cyclotron resonance the latter becoming higher than 150 - $200 \mathrm{keV}$ after 16:30 UT.

At higher energies above $250 \mathrm{keV}$, however, strong and lasting increases of parallel fluxes can be observed before 15:25 UT and after 16:30 UT, in an energy range unreachable by particles accelerated via Landau trapping. At 15 : $00-15: 25 \mathrm{UT}$, it stems probably

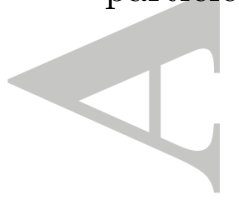

(C)2015 American Geophysical Union. All Rights Reserved. 
from the presence of fast magnetosonic waves measured at low latitudes, which accelerate electrons mainly above $300 \mathrm{keV}$ for $f_{p e} / f_{c e}<4.5$ [Mourenas et al., 2013]. Conversely, after 16:30 UT in Fig. 3j, it could be due to cyclotron resonant trapping acceleration or phase bunching of $200-500 \mathrm{keV}$ electrons by the measured low frequency quasi-parallel chorus waves, which could increase parallel fluxes or decrease perpendicular fluxes [Albert, 2002 , thereby increasing the ratio of parallel to perpendicular fluxes within this energy range. But a full description of flux variations at energies higher than $250 \mathrm{keV}$ is beyond the scope of this letter and left for future work.

\section{Discussion and conclusions}

Although quasi-linear models provide reasonable timescales for diffusive electron acceleration from $100 \mathrm{~s} \mathrm{keV}$ to $\mathrm{MeV}$ energies (with typically $\sim 10$ hours timescales) allowing to reproduce observations [Thorne et al., 2013], they often cannot explain a rapid acceleration from 1-10 keV to 100s keV due to faster losses [e.g., see Horne et al., 2005; Artemyev et al. 2015]. Thus, the problem of the seed $30-200 \mathrm{keV}$ population is still an area of active research. The Van Allen Probes mission allows for the first time to perform in situ coordinated and highly resolved measurements of magnetic and electric field waveforms, as well as detailed measurements of particle pitch-angle distributions over a wide energy range, providing the necessary basis for considering the effects of nonlinear wave-particle interactions in the radiation belts. The theory of nonlinear wave-particle interactions was originally developed by Nunn [1971]; Karpman and Shklyar [1972]. However, direct

experimental observations of this fine regime of wave-particle interaction are still lack-

ing. Here we have presented for the first time a clear experimental evidence of electron (C)2015 American Geophysical Union. All Rights Reserved. 
Landau resonant trapping into the electric field potential of oblique whistler-mode waves during a very long time interval (more than 6 hours), accompanied by an efficient and rapid acceleration (from $\sim 5-15 \mathrm{keV}$ up to $50-250 \mathrm{keV}$ ) of the trapped particles in the inhomogeneous geomagnetic field. The trapping process manifested itself by the formation of a plateau in the electron distribution at the energy of Landau resonance, as well as by a more field aligned trapped electron population. The energy range, density, and pitch-angle distribution of accelerated electrons are in good agreement with numerical simulations. Such a process operated continuously for more than 6 hours providing a seed population of about $100-200 \mathrm{keV}$ electrons. It suggests that this nonlinear acceleration mechanism may play an important role in the global dynamics of the outer radiation belt.

Acknowledgments. The work by O.A., F.M., and V.K. was performed under JHU/APL Contract No. 922613 (RBSP-EFW). A.V.A. is grateful to Dmitry Zimin Dynasty Foundation for support.

We also thank all the people associated with the electric field, magnetic field, and particle teams on the Van Allen Probes and the project team at the Johns Hopkins Applied Physics Laboratory that manages this program.

All data used in this paper can be found at RBSP/EFW database (http://www.space.umn.edu /missions/rbspefw-home-university-of-minnesota/)

\section{References}

Agapitov, O., A. Artemyev, V. Krasnoselskikh, Y. V. Khotyaintsev, D. Mourenas, H. Breuillard, M. Balikhin, and G. Rolland, Statistics of whistler mode waves in the (C)2015 American Geophysical Union. All Rights Reserved. 
outer radiation belt: Cluster STAFF-SA measurements, J. Geophys. Res., , 118, 34073420, doi:10.1002/jgra.50312, 2013.

Agapitov, O., A. Artemyev, D. Mourenas, V. Krasnoselskikh, J. Bonnell, O. Le Contel, C. M. Cully, and V. Angelopoulos, The quasi-electrostatic mode of chorus waves and electron nonlinear acceleration, J. Geophys. Res., , 119, 16061626, doi: 10.1002/2013JA019223, 2014a.

Albert, J. M., Nonlinear interaction of outer zone electrons with VLF waves, Geophys. Res. Lett., , 29, 1275, doi:10.1029/2001GL013941, 2002.

Albert, J. M., Simple approximations of quasi-linear diffusion coefficients, J. Geophys. Res., 112, A12202, doi:10.1029/2007JA012551, 2007.

Artemyev, A., V. Krasnoselskikh, O. Agapitov, D. Mourenas, and G. Rolland, Nondiffusive resonant acceleration of electrons in the radiation belts., Physics of Plasmas, 19, 122,901, doi:10.1063/1.4769726, 2012.

A.V. Artemyev, A.A. Vasiliev, D. Mourenas, O.V. Agapitov, V.V. Krasnoselskikh, Nonlinear electron acceleration by oblique whistler waves: Landau resonance vs. cyclotron resonance Physics of Plasmas (1994-present) 20 (12), 122901, 2013

Artemyev, A., O. Agapitov, F. Mozer, and V. Krasnoselskikh, Thermal electron acceleration by localized bursts of electric field in the radiation belts, Geophysical Research Letters, 41(16), 5734-5739, 2014a.

Artemyev, A., A. Vasiliev, D. Mourenas, O. Agapitov, and V. Krasnoselskikh, Electron scattering and nonlinear trapping by oblique whistler waves: The critical wave intensity for nonlinear effects, Physics of Plasmas (1994-present), 21(10), 102,903, 2014b.

(C)2015 American Geophysical Union. All Rights Reserved. 
Artemyev A. V., A. A. Vasiliev, D. Mourenas, O. V. Agapitov, V. Krasnoselskikh, D. Boscher and G. Rolland Fast transport of resonant electrons in phase space due to nonlinear trapping by whistler waves, Geoph. Res. Lett., DOI: 10.1002/2014GL061380, $2014 b$.

Artemyev, A. V., O. V. Agapitov, D. Mourenas, V. V. Krasnoselskikh, and F. S. Mozer, Wave energy budget analysis in the Earth's radiation belts uncovers a missing energy, Nature Communications, 6, 8143, doi:10.1038/ncomms8143, 2015.

Bell, T. F., The wave magnetic field amplitude threshold for nonlinear trapping of energetic gyroresonant and Landau resonant electrons by nonducted VLF waves in the magnetosphere, J. Geophys. Res., , 91, 4365-4379, doi:10.1029/JA091iA04p04365, 1986.

Blake, J. B., et al., The Magnetic Electron Ion Spectrometer (MagEIS) Instruments Aboard the Radiation Belt Storm Probes (RBSP) Spacecraft, Space Sci. Rev.,, 179, 383-421, doi:10.1007/s11214-013-9991-8, 2013.

Bortnik, J., R. M. Thorne, and U. S. Inan, Nonlinear interaction of energetic electrons with large amplitude chorus, Geophys. Res. Lett., , 35, L21102, doi:10.1029/2008GL035500, 2008.

Cattell, C., et al., Discovery of very large amplitude whistler-mode waves in Earth's radiation belts, Geophys. Res. Lett., , 35, L01105, doi:10.1029/2007GL032009, 2008.

Cattell, C. A., A. W. Breneman, S. A. Thaller, J. R. Wygant, C. A. Kletzing, and W. S. Kurth, Van Allen Probes observations of unusually low frequency whistler-mode waves observed in association with moderate magnetic storms: Statistical Study, Geophys.

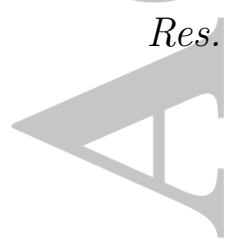
Res. Lett., , 42, L7273-7281, doi:10.1002/2015GL065565, 2015. 
Cully, C. M., J. W. Bonnell, and R. E. Ergun, THEMIS observations of long-lived regions of large-amplitude whistler waves in the inner magnetosphere, Geophys. Res. Lett., , 35, L17S16, doi:10.1029/2008GL033643, 2008.

Drake, J., O. Agapitov, and F. Mozer, The development of a bursty precipitation front with intense localized parallel electric fields driven by whistler waves, Geophysical Research Letters, 42(8), 2563-2570, 2015.

Funsten, H. O., et al., Helium, Oxygen, Proton, and Electron (HOPE) Mass Spectrometer for the Radiation Belt Storm Probes Mission, Space Sci. Rev.,, 179, 423-484, doi: 10.1007/s11214-013-9968-7, 2013.

Gendrin, R., Pitch angle diffusion of low energy protons due to gyroresonant interaction with hydromagnetic waves, Journal of Atmospheric and Terrestrial Physics, 30, 13131330, doi:10.1016/S0021-9169(68)91158-6, 1968.

Glauert, S. A., and R. B. Horne, Calculation of pitch angle and energy diffusion coefficients with the PADIE code, J. Geophys. Res., 110, A04206, doi:10.1029/2004JA010851, 2005.

Horne, R. B., et al., Timescale for radiation belt electron acceleration by whistler mode chorus waves, J. Geophys. Res., 110, A03225, doi:10.1029/2004JA010811, 2005.

Horne, R. B., et al., Wave acceleration of electrons in the van allen radiation belts, Nature, $437(7056), 227-230,2005$.

Karpman, V. I., and D. R. Shklyar, Nonlinear Damping of Potential Monochromatic Waves in an Inhomogeneous Plasma, Sov. JETP, 35, 500, 1972.

Kellogg, P. J., C. A. Cattell, K. Goetz, S. J. Monson, and L. B. Wilson, III, Electron trapping and charge transport by large amplitude whistlers, Geophys. Res. Lett., , 37,

(C)2015 American Geophysical Union. All Rights Reserved. 
L20106, doi:10.1029/2010GL044845, 2010.

Kellogg, P. J., C. A. Cattell, K. Goetz, S. J. Monson, and L. B. Wilson, III, Large amplitude whistlers in the magnetosphere observed with Wind-Waves, J. Geophys. Res., , 116, A09224, doi:10.1029/2010JA015919, 2011.

Kennel, C. F., and H. E. Petschek, Limit on Stably Trapped Particle Fluxes, J. Geophys. Res., , 71, 1-28, 1966.

Kletzing, C. A., et al., The Electric and Magnetic Field Instrument Suite and Integrated Science (EMFISIS) on RBSP, Space Sci. Rev.,, 179, 127-181, doi:10.1007/s11214-0139993-6, 2013.

Lyons, L. R., R. M. Thorne, and C. F. Kennel, Pitch-angle diffusion of radiation belt electrons within the plasmasphere., J. Geophys. Res., , 7r, 3455-3474, doi: 10.1029/JA077i019p03455, 1972.

Malaspina, D. M., L. Andersson, R. E. Ergun, J. R. Wygant, J. W. Bonnell, C. Kletzing, G. D. Reeves, R. M. Skoug, and B. A. Larsen, Nonlinear electric field structures in the inner magnetosphere, Geophys. Res. Lett., , 41, 5693-5701, doi:10.1002/2014GL061109,

\section{4.}

Min, K., K. Liu, and W. Li, Signatures of electron landau resonant interactions with chorus waves from themis observations, Journal of Geophysical Research: Space Physics, $119(7), 5551-5560,2014$.

Mourenas, D., A. Artemyev, O. Agapitov, and V. Krasnoselskikh, Consequences of geomagnetic activity on energization and loss of radiation belt electrons by oblique chorus waves, Journal of Geophysical Research: Space Physics, 119(4), 2775-2796, 2014a.

(C)2015 American Geophysical Union. All Rights Reserved. 
Mourenas, D., A. Artemyev, O. Agapitov, V. Krasnoselskikh, and F. Mozer, Very oblique whistler generation by low energy electron streams, Journal of Geophysical Research: Space Physics, 2015.

Mourenas, D., A. V. Artemyev, O. V. Agapitov, and V. V. Krasnoselskikh, Analytical estimates of electron quasi-linear diffusion by fast magnetosonic waves, J. Geophys. Res., 118, 3096-3112, doi:10.1002/jgra.50349, 2013.

Mozer, F., O. Agapitov, V. Krasnoselskikh, S. Lejosne, G. Reeves, and I. Roth, Direct observation of radiation-belt electron acceleration from electron-volt energies to megavolts by nonlinear whistlers, Physical review letters, $113(3), 035,001,2014$.

Mozer, F., O. Agapitov, A. Artemyev, J. Drake, V. Krasnoselskikh, S. Lejosne, and I. Vasko, Time domain structures: what and where they are, what they do, and how they are made, Geophysical Research Letters, 2015.

Nunn, D., Wave-particle interactions in electrostatic waves in an inhomogeneous medium, Journal of Plasma Physics, 6, 291, doi:10.1017/S0022377800006061, 1971.

Nunn, D., and Y. Omura, A computational and theoretical investigation of nonlinear waveparticle interactions in oblique whistlers, Journal of Plasma Physics, 120, 28902911, doi:10.1002/2014JA020898, 2015.

Omura, Y., N. Furuya, and D. Summers, Relativistic turning acceleration of resonant electrons by coherent whistler mode waves in a dipole magnetic field, J. Geophys. Res., 112, A06236, doi:10.1029/2006JA012243, 2007.

Reeves, G. D., et al., Electron Acceleration in the Heart of the Van Allen Radiation Belts, Science, 341, 6149, doi:10.1126/science.1237743, 2013.

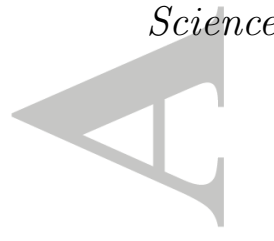

(C)2015 American Geophysical Union. All Rights Reserved. 
Schulz, M., and L. J. Lanzerotti, Particle diffusion in the radiation belts, 1974.

Shklyar, D., and H. Matsumoto, Oblique Whistler-Mode Waves in the Inhomogeneous Magnetospheric Plasma: Resonant Interactions with Energetic Charged Particles, Surveys in Geophysics, 30, 55-104, doi:10.1007/s10712-009-9061-7, 2009.

Shprits, Y. Y., N. P. Meredith, and R. M. Thorne, Parameterization of radiation belt electron loss timescales due to interactions with chorus waves, Geophys. Res. Lett., , 34, L11110, doi:10.1029/2006GL029050, 2007.

Spence, H. E., et al., Science Goals and Overview of the Radiation Belt Storm Probes (RBSP) Energetic Particle, Composition, and Thermal Plasma (ECT) Suite on NASA's Van Allen Probes Mission, Space Sci. Rev.,, 179, 311-336, doi:10.1007/s11214-0130007-5, 2013.

Summers, D., Y. Omura, S. Nakamura, and C. A. Kletzing, Fine structure of plasmaspheric hiss, J. Geophys. Res., , 119, 91349149, doi:10.1002/2014JA020437, 2014.

Thorne, R. M., B. Ni, X. Tao, R. B. Horne, and N. P. Meredith, Scattering by chorus waves as the dominant cause of diffuse auroral precipitation, Nature, , 467, 943-946, doi:10.1038/nature09467, 2010.

Thorne, R. M., et al., Rapid local acceleration of relativistic radiation-belt electrons by magnetospheric chorus, Nature,, 504, 411-414, doi:10.1038/nature12889, 2013.

Trakhtengerts, V. Y., Stationary states of the Earth's outer radiation zone, Geomagnetism and Aeronomy, 6, 827-836, 1966.

Vasko, I., O. Agapitov, F. Mozer, and A. Artemyev, Thermal electron acceleration by electric field spikes in the outer radiation belt: generation of field-aligned pitch angle

(C)2015 American Geophysical Union. All Rights Reserved. 
distributions, Journal of Geophysical Research: Space Physics, 2015.

Wilson, L. B., III, C. A. Cattell, P. J. Kellogg, J. R. Wygant, K. Goetz, A. Breneman, and K. Kersten, The properties of large amplitude whistler mode waves in the magnetosphere: Propagation and relationship with geomagnetic activity, Geophys. Res. Lett., , 38, L17107, doi:10.1029/2011GL048671, 2011.

Wygant, J. R., et al., The Electric Field and Waves Instruments on the Radiation Belt Storm Probes Mission, Space Sci. Rev., 179, 183-220, doi:10.1007/s11214-013-0013-7,
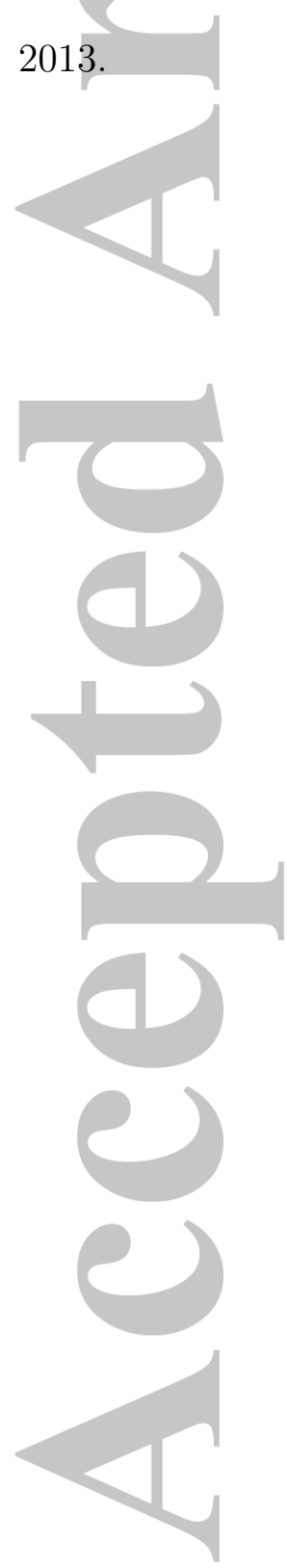

(C)2015 American Geophysical Union. All Rights Reserved. 

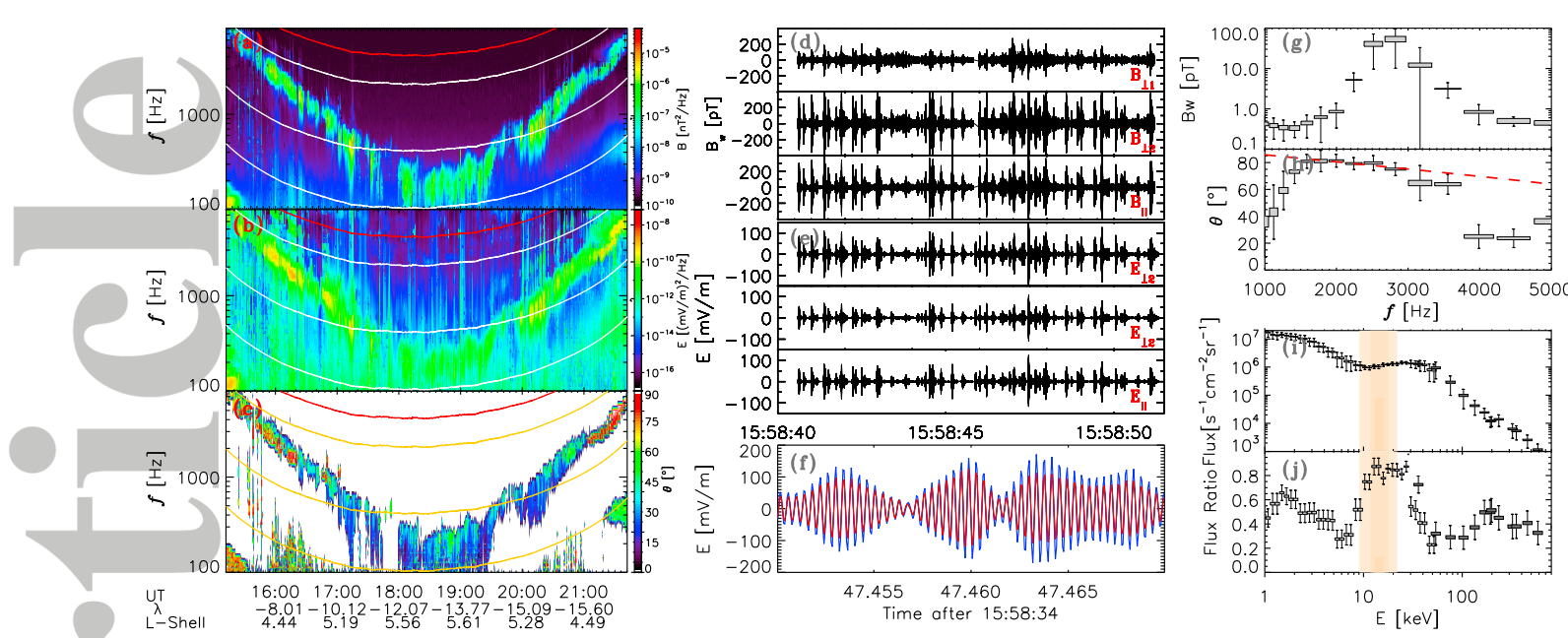

Figure 1. Observations of oblique chorus waves and corresponding effects in electron fluxes collected aboard Van Allen Probe B on November 1, 2012. (a,b) magnetic and electric field spectrograms from EMFISIS measurements (lower hybrid frequency $f_{L H}, 0.1 f_{c e}, 0.5 f_{c e}$ based on in situ magnetic field measurements are indicated by white lines and $f_{c e}$ by red line); panel (c) shows the wave normal angle $\theta$. Three component waveforms (in the magnetic field aligned coordinate system) of magnetic and electric fields captured by EFW at 15:58 UT during the period of enhanced electric field fluctuations are presented in panels (d) and (e) respectively. Panel (f) shows $20 \mathrm{~ms}$ zoom on the electric field perturbation waveform presented in panel (e), the parallel $E_{w}$ component being marked by red color. Panels (g) and (h) show, respectively, the magnetic field wave power spectrum integrated in each EMFISIS channel and the wave normal angle $\theta$ at 15:58 UT (the red dashed line indicates the value of the whistler-mode resonant cone angle $\theta_{r}$. Panels (i) and (j) present parallel electron fluxes captured at 15:58 UT by HOPE and MagEIS and the parallel over transverse flux ratio (relative to the local background magnetic field direction). The energy range highlighted in red corresponds to the full width of the Landau resonance (see text for details).

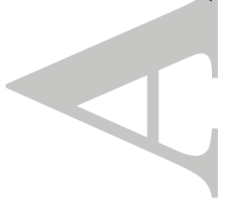

(C)2015 American Geophysical Union. All Rights Reserved. 

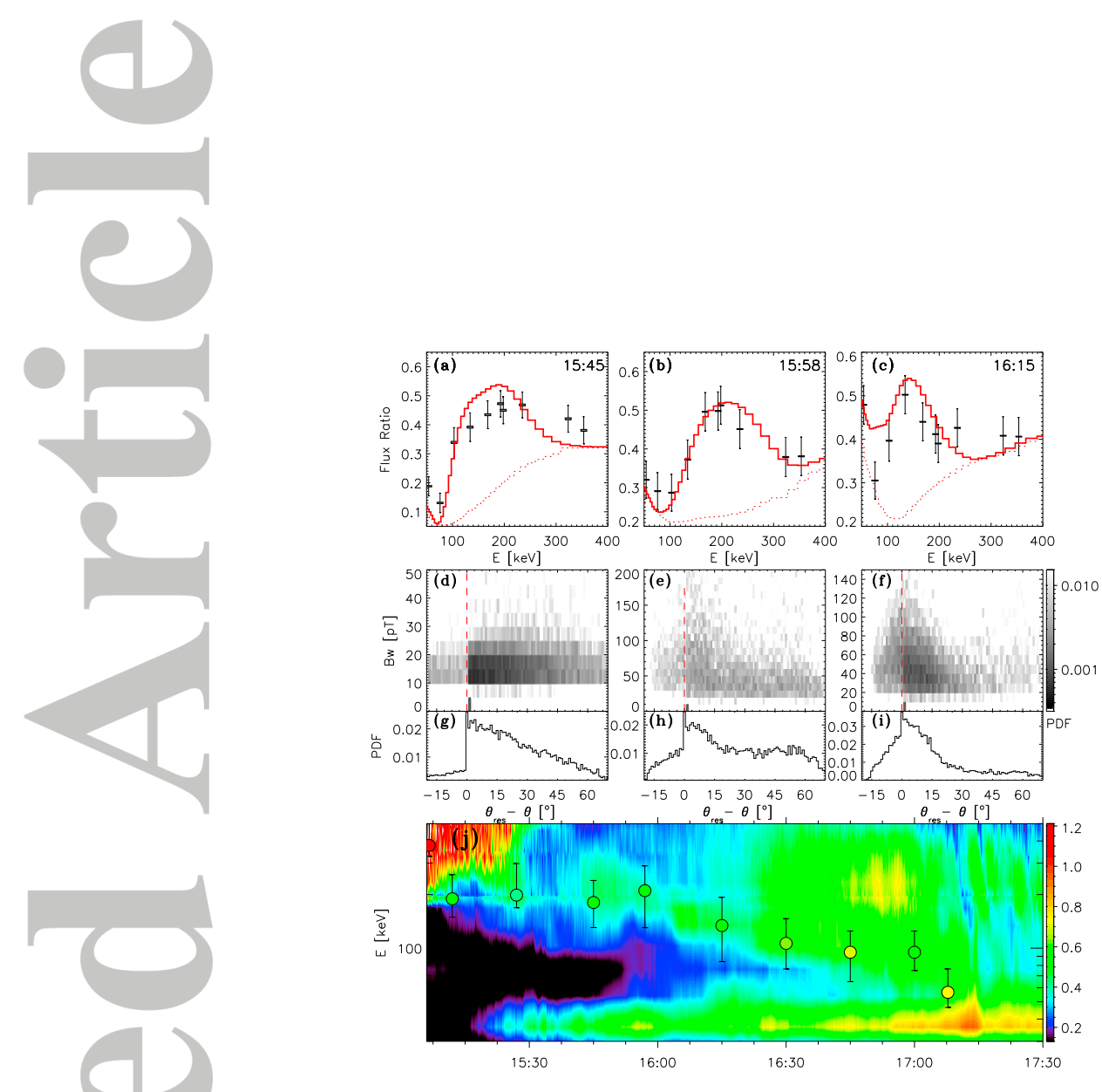

Figure 2. (a) Field aligned to transverse electron flux ratio as a function of electron energy and time. (b) Ratio of the parallel electron flux over the parallel flux value in the lower neighboring energy channel. (c) Resonant energy of electrons calculated for Landau trapping conditions from the measured parameters of whistler-mode waves (the wave frequency in $\mathrm{kHz}$ is indicated by color and wave electric field amplitude by dot size).

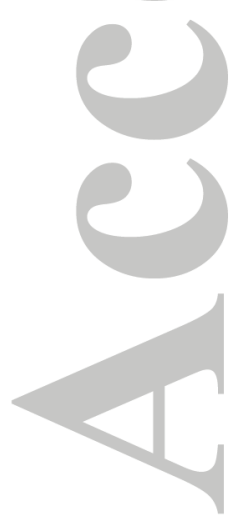

(C)2015 American Geophysical Union. All Rights Reserved. 

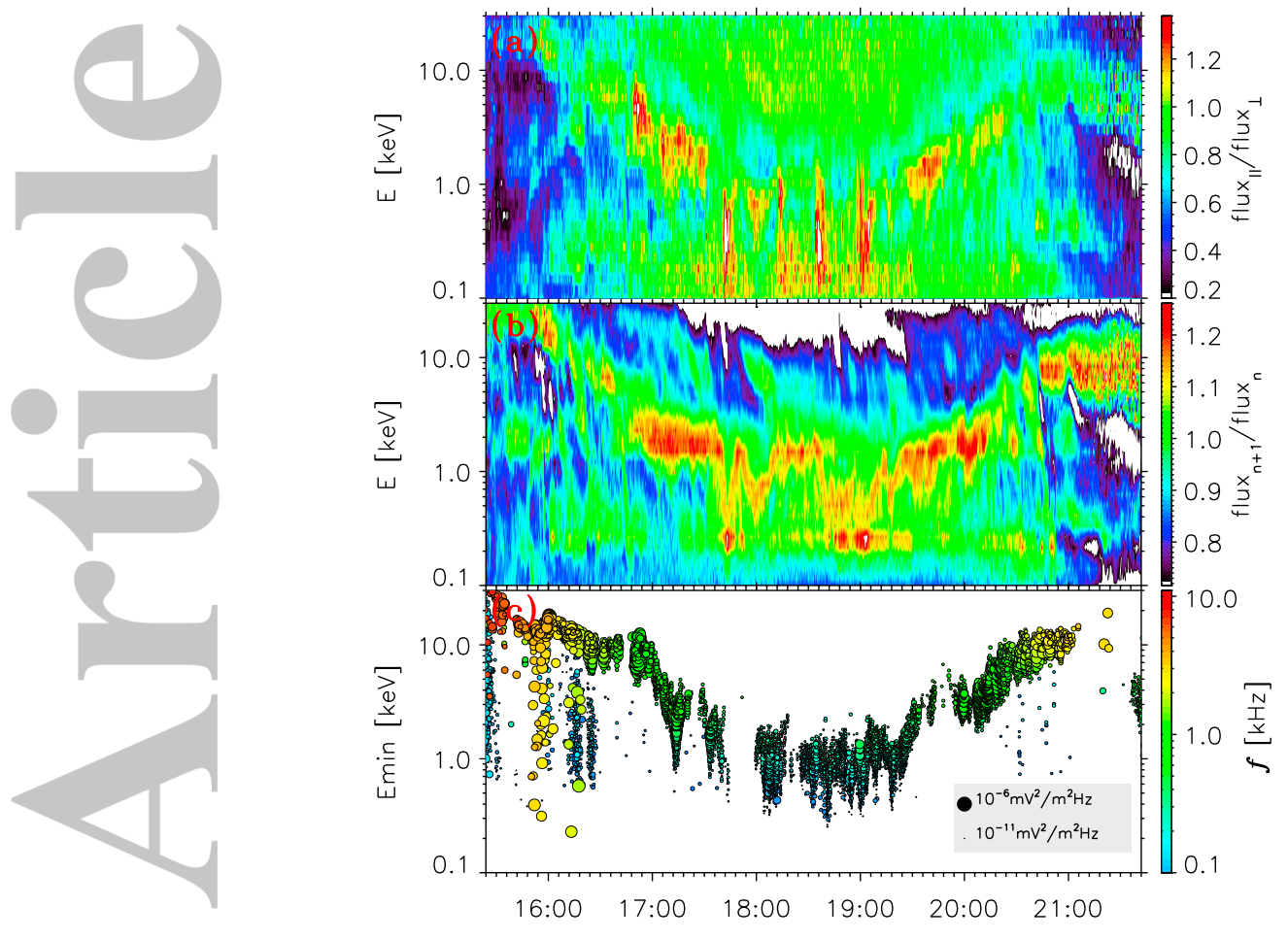

Figure 3. Field aligned to transverse electron flux ratio at three different times: 15:45 UT (a), 15:58 UT (b) (see details concerning this case in Fig.1) and 16:15 UT (c). Numerical results of test particle simulations are displayed in red, while corresponding MagEIS observations are denoted by black crosses (dashed curves showing approximate fits to the initial flux ratio measured 5-10 min before). The corresponding chorus wave distributions from EMFISIS used in the numerical simulations are displayed in panels $(\mathrm{d}, \mathrm{e}, \mathrm{f})$ in the $\left(B_{w}\right)-\left(\theta-\theta_{r}\right)$ domain and in panels $(\mathrm{g}, \mathrm{h}, \mathrm{i})$ under the form of a probability distribution function as a function of $\left(\theta-\theta_{r}\right)$. A global comparison between the ratio of field aligned over transverse electron fluxes obtained from MagEIS measurements with numerical results is presented in panel $(\mathrm{j})$. Colored circles indicate the energy of the distribution maximum, with colors corresponding to the maximum value of the flux ratio (see panels a,b,c) and distribution widths shown by error bars.

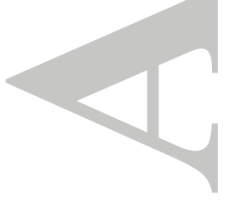

(C)2015 American Geophysical Union. All Rights Reserved. 